\title{
O Programa Articuladores da Atenção Básica: uma proposta inovadora para qualificação da Atenção Básica
}

\author{
The Articulator of Primary Health Care Program: \\ an innovative proposal for qualification of Primary Health Care
}

Giovanna Cabral Doricci ${ }^{1}$

Carla Guanaes-Lorenzi ${ }^{1}$

Maria José Bistafa Pereira ${ }^{2}$
${ }^{1}$ Departamento de Psicologia, Faculdade de Filosofia Ciências e Letras de Ribeirão Preto, Universidade de São Paulo (USP). Av. Bandeirantes 3900, Monte Alegre. 14040-901 Ribeirão Preto SP Brasil. psicologia. dorigica@gmail.com ${ }^{2}$ Departamento de Enfermagem MaternoInfantil e Saúde Pública, Escola de Enfermagem de Ribeirão Preto, USP Ribeirão Preto SP Brasil.
Abstract In 2009, the Secretary of State for Health of Sao Paulo created a Program with a view to qualify the primary care in the state. This proposal includes a new job function, namely the articulator of primary care. Due to the scarcity of information about the practice of these new professionals in the scientific literature, this article seeks to analyze how articulators interpret their function and how they describe their daily routines. Thirteen articulators were interviewed. The interviews were duly analyzed by qualitative delineation. The results describe three themes: 1) Roles of the articulator: technical communicator and political advisor; 2) Activities performed to comply with the expected roles, examples being diagnosis of the municipalities, negotiation of proposals, participation in meetings, visits to municipalities; and 3) Challenges of the role, which are configured as challenges to the health reform process, examples being the lack of physical and human resources, activities of professionals in the medical-centered model, among others. The conclusion drawn is that the Program has great potential to provide input for the development and enhancement of Primary Care. Nevertheless, there are a series of challenges to be overcome, namely challenges to the context per se.

Key words Primary health care, Health management, Technical cooperation
Resumo A Secretaria Estadual da Saúde de São Paulo criou em 2009 um Programa com o objetivo de qualificar a atenção básica do estado. Esta proposta inclui uma nova função profissional chamada articulador da atenção básica. Devido à escassez de informações na literatura científica a respeito da prática destes novos profissionais, este artigo tem como objetivo analisar o modo como os articuladores significam sua função e descrevem sua atuação cotidiana. Foram realizadas 13 entrevistas com articuladores e analisadas por delineamento qualitativo. Os resultados descrevem três temas: 1) Papéis do articulador: assessor técnico, comunicador e político; 2) Atividades desempenhadas para cumprir os papéis esperados, são exemplos: diagnóstico dos municípios, negociação de propostas, participação de reuniões, visitas aos municípios; e, 3) Desafios da função que se configuram como desafios do próprio processo da Reforma Sanitária, são exemplos: a falta de recursos físicos e humanos, atuação dos profissionais em modelo médico-centrado, dentre outros. Concluimos que este Programa possui importante potencial para pensarmos sobre o desenvolvimento e o fortalecimento da Atenção Básica. Por outro lado, há uma série de desafios para serem superados, desafios do próprio contexto.

Palavras-chave Atenção básica, Gestão em saúde, Cooperação técnica 


\section{Introdução}

Desde 1978, com a Conferência Internacional de Alma Ata, os cuidados primários passaram a ser considerados estratégia fundamental para a promoção das Reformas Sanitárias em vários países, dentre eles o Brasil. Aos poucos, após instituição do Sistema Único de Saúde (SUS) em 1988, marco histórico da Reforma Sanitária, os cuidados primários foram ganhando destaque através de Programas, como o dos Agentes Comunitários de Saúde e o Programa de Saúde da Família, os quais atualmente fazem parte da Política Nacional de Atenção Básica de Saúde (PNAB) regulamentada desde $2006^{1}$. Neste artigo, conforme Portaria ${ }^{1}$, as nomenclaturas Atenção Básica (AB) e Atenção Primária em Saúde (APS) são utilizadas como sinônimos, embora consideremos haver diferenças práticas e filosóficas em suas origens.

Trinta anos após a Conferência de Alma Ata, a Organização Mundial de Saúde (OMS) reiterou a importância dos cuidados primários e fomentou estudos e discussões sobre este tema ${ }^{2,3}$. Da mesma forma, este resgate fez parte da agenda da Organização Pan-Americana de Saúde nos anos $2000^{4}$. Assim, a temática dos cuidados primários e seu potente papel reorganizador dos sistemas de saúde se mantêm vivos e em constante debate, nacional e internacional, em que são destacadas a necessidade de fortalecimento de suas potencialidades, e, ao mesmo tempo, de superação de seus desafios ${ }^{5-10}$.

Dentre os principais desafios nacionais e internacionais ${ }^{2,3,9,10}$ para a consolidação da $A B$ destacados na literatura estão as necessidades de: aperfeiçoamento dos processos de monitoramento e avaliação com base em indicadores; auxílio às equipes no manejo dos indicadores; aperfeiçoamento da capacidade da gestão dos coordenadores; informatização; e reforma das unidades existentes ${ }^{8}$. Em muitos casos, o trabalho em equipe multiprofissional ainda se mantém fragmentado, o que prejudica a assistência integral; há desarticulação no que diz respeito à referência e contrarreferência; a formação dos profissionais ainda se mantém no modelo biomédico; as condições de trabalho, em muitos lugares, são precárias, havendo grande instabilidade, baixos salários, jornada excessiva e escassez de recursos ${ }^{7}$.

Considerando estes, e muitos outros desafios destacados na literatura ${ }^{11-13}$, diversas iniciativas têm sido criadas com o objetivo de qualificar e fortalecer a $\mathrm{AB}$, como, por exemplo, os Núcleos de Apoio à Saúde da Família (NASF), o Programa Requalifica UBS, o Programa Nacional de Me- lhoria do Acesso e da Qualidade da Atenção Básica (PMAQ-AB). Estas propostas do Ministério da Saúde são negociadas em nível regional, onde as pactuações intermunicipais são realizadas. Além destas estratégias federais, conforme apontam Almeida et al. ${ }^{13}$, há estratégias de fortalecimento da atenção básica que são estabelecidas nos níveis municipal e regional de acordo as especificidades de cada local.

Considerando este movimento e essa necessidade de qualificação da $\mathrm{AB}$ presente no discurso oficial das políticas de saúde do país ${ }^{5}$, em 2009 a Secretaria Estadual da Saúde do estado de São Paulo (SES-SP) lançou um Programa com o objetivo de qualificar a $\mathrm{AB}$ através do apoio técnico oferecido à gestão municipal. A proposta incluiu a criação de uma nova função profissional, a do articulador da atenção básica, para assessorar gestores e profissionais de saúde desses municípios a analisar a efetividade da $\mathrm{AB}$, e, a partir das necessidades de cada região, incluir propostas de mudança (que vão desde a adesão de programas federais a propostas e pactuações locais) em prol de sua qualificação.

Este novo Programa configura-se como experiência relacionada ao processo de descentralização (gestão cooperativa entre estado e município) e à necessidade de políticas que efetivem a $\mathrm{AB}$ como ordenadora do SUS, proposta que também acompanha o debate internacional ${ }^{11-13}$. Portanto, os acontecimentos históricos que criaram a necessidade dessa iniciativa estão relacionados às transformações ocorridas na gestão, através dos processos de descentralização e regionalização, além da necessidade de qualificação da $\mathrm{AB}^{14}$.

Durante os anos noventa, os estados permaneceram afastados da gestão ${ }^{11,12,15}$. A Norma Operacional de Assistência à Saúde, NOAS 01/02, procurou modificar o foco da municipalização autárquica à regionalização ${ }^{16}$ através do Plano Diretor de Regionalização (PDR) que alterou também a gestão envolvendo de maneira mais direta a participação dos estados ${ }^{17}$. Atualmente, as diretrizes de operacionalização da descentralização e regionalização são contempladas pelo Pacto Pela Saúde 2006. De acordo com o Pacto de Gestão, que corresponde a uma das dimensões do Pacto Pela Saúde, a gestão passou a ser baseada na cooperação entre estados e municípios, e a regionalização passou a ser estabelecida com base em territórios sanitários e não político-administrativos ${ }^{18}$.

A partir dessas mudanças surgiu um novo formato de gestão estadual, que prevê a participação ativa das Secretarias Estaduais de Saúde 
(SES) nas formulações políticas e na oferta de apoio técnico à gestão municipal. Devido à nova configuração da gestão, o Plano Estadual de Saúde (2008-2011) definiu o fortalecimento da $\mathrm{AB}$ como prioridade para o estado de São Paulo. Com esse objetivo, a SES-SP, em parceria com as universidades UNESP (Universidade Estadual Paulista) e UNICAMP (Universidade Estadual de Campinas), lançou um programa inovador, o Programa Articuladores da Atenção Básica, que pode ser considerado como "[...] uma política de apoio do estado para os municípios, priorizando aqueles com população inferior a 100 mil habitantes, com o objetivo de auxiliar os gestores municipais na qualificação dos serviços de Atenção Básica e de fortalecer esse nível de atenção [... $]^{\text {"14 }}$.

Esse novo projeto foi regulamentado inicialmente através da Resolução SS - 187 de 05 de Dezembro de $2008^{19}$, posteriormente revogada e substituída pela Resolução SS - 61 de 14 de Junho de 2011. Esta resolução institui a função do articulador da atenção básica e descreve, em linhas gerais, o objetivo do Programa de monitorar e avaliar o desempenho e a evolução da $\mathrm{AB}$ no estado, e propor ações de intervenção e capacitação em $\mathrm{AB}$ que sejam pactuadas. No entanto, não descreve quais são as atividades a serem realizadas pelos articuladores. $\mathrm{O}$ que se espera, em termos práticos, desta nova função profissional? Quais são as atividades desempenhadas na prática desta função?

No site da Secretaria Estadual, o Programa é definido como "serviço de apoio técnico [...] que envolve avaliação, monitoramento e apoio ao desenvolvimento da gestão municipal da atenção básica, atividade [...] desenvolvida conforme orientação técnico-normativa" ${ }^{20}$. Essa descrição é bastante genérica e não inclui as atividades que estes profissionais deverão desempenhar no exercício de sua função.

O primeiro processo seletivo para contratação dos articuladores incluiu a análise do currículo e discussões temáticas para a confecção de trabalhos avaliados como parte do processo. Os articuladores selecionados foram distribuídos de acordo com as Regiões de Saúde estabelecidas em 2007, após adesão do estado ao Pacto Pela Saúde ${ }^{14}$. O estado de São Paulo possui 64 Regiões de Saúde geridas pelos Colegiados de Gestão Regional (CGR). O conjunto das Regiões de Saúde compõe as Regionais de Saúde que estão sob gestão dos Departamentos Regionais de Saúde (DRS). O estado está dividido em $17 \mathrm{DRS}^{19}$. Os articuladores fazem parte do quadro de profissionais da SES-SP ${ }^{14}$, mas estão subordinados admi- nistrativamente às $\mathrm{DRS}^{21}$. A proporção média de distribuição dos articuladores é de 1 para cada 10 municípios de uma mesma Região ${ }^{14}$.

Andrade e Castanheira ${ }^{14}$ descrevem a implantação do Programa, entre 2009 e 2010, em dois momentos: visita aos municípios, estratégia inicial em que os articuladores tomaram um primeiro contato com a realidade local e realizaram um diagnóstico através de um roteiro dirigido; e desenvolvimento de planos de trabalho por meio de oficinas mensais com o objetivo de levantar propostas frente às necessidades observadas nos diagnósticos realizados.

Com base em revisão de literatura é possível concluir que há uma escassez de estudos sobre este Programa no estado de São Paulo. As informações localizadas no site da Secretaria Estadual de Saúde sobre a função dos articuladores são ainda genéricas, o que sugere que ela tem sido construída pelos profissionais em sua prática cotidiana. O Programa parece ser pouco divulgado e pouco conhecido até mesmo no estado de São Paulo onde foi criado. Porém, essa experiência construída especificamente no estado de São Paulo, pode constituir importante recurso para qualificação da $\mathrm{AB}$ que corresponde ao modelo de atenção à saúde adotado, dentre outras propostas, como fundamental para concretização da Reforma Sanitária do país.

Como colocado, parte da fundamentação da Reforma Sanitária do Brasil decorre do movimento internacional de enfoque aos cuidados primários lançado pela OMS a partir de 1978 e ainda em desenvolvimento. Assim, embora o Programa dos articuladores, em princípio, apareça como parte de uma política estadual, sua importância ultrapassa as fronteiras estaduais e nacionais revelando-se profícuo ao contribuir, também, com o debate internacional de construção e qualificação da APS.

A partir desta fundamentação introdutória, e da lacuna reconhecida na literatura científica, o presente artigo tem como objetivo descrever e analisar o modo como os articuladores da atenção básica significam sua função.

\section{Método}

Neste artigo, relatamos um estudo qualitativo, descritivo e exploratório, que foi desenvolvido com treze articuladores, todos de uma das Redes Regionais de Atenção à Saúde de São Paulo (RRAS). Esta Rede foi escolhida por ser a mais próxima, geograficamente, do local de realização 
da pesquisa. A idade dos participantes está entre 37 e 60 anos, sendo 2 do sexo masculino e 11 do feminino. Possuem formação acadêmica em Psicologia, Medicina, Enfermagem ou Odontologia. Para manter sigilo quanto à identidade dos profissionais buscamos conservar a palavra "articulador" sempre no gênero masculino.

Utilizamos como instrumento uma entrevista semiestruturada com foco em quatro assuntos principais: Caracterização geral dos participantes; A implantação do Programa; Descrição da prática atual; e Avaliação dos articuladores sobre o Programa. As conversas foram realizadas no primeiro semestre de 2013, gravadas em áudio e posteriormente transcritas na íntegra.

A pesquisa possui delineamento qualitativo de caráter construcionista social. O objetivo da pesquisa pautada neste delineamento é compreender como as pessoas produzem sentidos sobre o mundo em que vivem, e refletir sobre as práticas legitimadas por esses modos de compreensão ${ }^{22,23}$.

Utilizamos em nossa análise os conceitos de produção de sentidos e práticas discursivas ${ }^{24}$. Como definem Spink e Medrado ${ }^{25}$, "a produção de sentido [...] é uma prática social, dialógica, que implica a linguagem em uso [...] é tomada, portanto, como um fenômeno sócio-lingüístico (sic) ${ }^{\prime 25}$. A ideia principal é a de que a prática resulta dos sentidos construídos em um processo dialógico e situado - social, histórica e culturalmente.

Nesta abordagem teórico-metodológica, o conteúdo das falas é considerado como resultado de uma construção conjunta, que decorre da relação entre pesquisador e participante ${ }^{26}$. Portanto, a análise busca explicitar os diversos sentidos que se tornaram possíveis no processo conversacional, não havendo preocupação em avaliar a frequência de aparecimento dos mesmos.

Podemos descrever os seguintes passos da análise: 1) Transcrições integrais das entrevistas, de acordo com a adaptação das regras descritas por Preti ${ }^{27}$; 2) Leitura em profundidade guiada pelo tema central e objetivo do estudo quanto à prática da função dos articuladores; 3) Construção temática e descrição dos sentidos mediante o confronto dos conhecimentos prévios do pesquisador e de suas teorias de base com as informações produzidas em sua relação com os participantes $^{28}$.

A pesquisa acatou os parâmetros éticos para o desenvolvimento de estudos com seres humanos tendo sido aprovada pelo Comitê de Ética. Os participantes assinaram o Termo de Consentimento Livre e Esclarecido, e tiveram seus nomes omitidos na divulgação dos resultados.

\section{Resultados e discussão}

Os resultados apresentados descrevem o modo como os articuladores significam sua função e atuação, a partir de três temas: 1) Papéis do Articulador; 2) Atividades Desempenhadas; e 3 ) Desafios. O termo "papel”, conforme definimos no estudo, refere-se aos objetivos da função, e as "atividades" são as ações desempenhadas para atingir essas metas. Ao longo da análise apresentamos alguns excertos das entrevistas com o objetivo de ilustrar os sentidos produzidos em torno destas temáticas.

\section{Papéis do articulador}

O Quadro 1 organiza os principais sentidos construídos sobre os papéis desempenhados pelos articuladores da atenção básica.

Um dos papéis destacados pelos articuladores é o de "assessoria técnica", que implica ofertar apoio, orientação e informação tanto para as equipes (administrativas e assistenciais) quanto para os gestores dos municípios pelos quais são responsáveis.

E1: então, é realmente assim, assessorar aquilo que eles, que eles sentem necessidade, de repente a gente está fazendo coisa que nem é atenção básica [...] eles ligam pra gente.

Apesar de terem sido criados para qualificar a $\mathrm{AB}$, os articuladores acabam ofertando auxílio aos municípios seja o assunto abordado referente à $\mathrm{AB}$ ou não. Considerando a proposta da $\mathrm{AB}$ como ordenadora do SUS ${ }^{6,29}$, essa atuação ampliada dos articuladores se mostra necessá-

Quadro 1. Papéis do Articulador.

\begin{tabular}{|c|l|}
\hline Tema & \multicolumn{1}{c|}{ Sentidos } \\
\hline Papéis / Objetivo da função & - Assessoraria técnica; \\
& - Ampliação da comunicação interinstitucional; \\
& - Papel político - defesa de um modelo de atenção pautado em AB ampliada. \\
\hline
\end{tabular}


ria para que se torne possível a integralidade da atenção, a conformação das redes de cuidado e da intersetorialidade. Por outro lado, a abertura para atuar em todos os contextos do sistema pode gerar uma sobrecarga de trabalho, em que os articuladores passam a ser vistos como referência independente do assunto.

Outro papel destacado é o de facilitador da comunicação entre as instituições que representam os entes federativos e profissionais que atuam na ponta do Sistema, como, por exemplo, a comunicação entre a SES e municípios (gestão e equipe); ou entre DRS e municípios (gestão e equipe), ou até mesmo entre o gestor municipal e suas equipes.

E10: eu acho que nós articuladores somos as pessoas que mais conseguem juntar as pessoas aqui [...] a gente consegue tanto ser esse elo de ligação entre gestão e município como também ser um elo de ligação entre a regional, intra-regional mesmo, e da regional com eles.

Um dos principais desafios apontados na literatura para que a APS seja de fato ordenadora do sistema decorre das dificuldades de comunicação existente entre os profissionais, setores, unidades de saúde, municípios, dentre outras instâncias ${ }^{29}$. Como afirmam Santos e Giovanella ${ }^{11}$, a carência de instrumentos que gerem a integração dos entes federados dificulta a coordenação dos níveis para a promoção de uma clínica efetiva. Diante deste cenário, a função dos articuladores pode ser considerada instrumento potencial para gerar esta integração, uma vez que estão em contato direto com os níveis municipal, regional e estadual.

Os articuladores destacam, ainda, um "papel político", que se refere à defesa de um modelo de atenção específico, através de seu posicionamento perante os erros ou as falhas que encontram no cotidiano das ações e serviços quando comparados às diretrizes políticas estabelecidas. Assim, como referido por Andrade e Castanheira ${ }^{14}$ este papel é político por compreender uma postura atenta nessa relação que estabelece com seus municípios.

E1: é... ele tem que ter um senso muito crítico em relação a política, certo? Não nós, nós não somos políticos, nós não nos envolvemos em política [...] todo mundo é político, na realidade, mas a gente não faz política partidária, a gente faz política de saúde pra melhorar aquilo que está precisando.

Portanto, a atuação do articulador deve estar pautada na promoção de mudanças a favor do enfoque na APS. Conforme consta na resolução que dispõe sobre o Programa ${ }^{21}$, seu objetivo é monitorar e avaliar a evolução da $\mathrm{AB}$ no estado. Para isso, oferece orientação técnica, e/ou, mais do que isso, procura sensibilizar gestores e equipes para que eles se sintam motivados e responsáveis pelas transformações propostas. Esse modo de atuação possibilita ao gestor compreender a importância e a utilidade do investimento nos cuidados primários, e às equipes a considerarem a importância de sua atuação neste processo, podendo inclusive sensibilizar a população quanto à nova proposta.

A partir deste enfoque, menos normativo e mais reflexivo, o articulador pode gerar movimentos iniciais, ao menos em nível local, de transformação cultural, em que a $\mathrm{AB}$ possa ser compreendida em seu caráter ampliado ${ }^{5,29}$ e não mais como pacotes básicos de assistência para a população carente ${ }^{13}$, mas, para isso, o próprio articulador precisa ter uma compreensão ampliada da AB.

Fundamentados pelos papéis definidos em nossa análise, compreendemos que a função do articulador tem como objetivo central apreender as necessidades locais e informar sobre as possibilidades de qualificação disponíveis no formato de políticas e programas criados pelo Ministério da Saúde, o que pode promover o alinhamento entre as propostas dos entes federativos e a indução de mudanças que se façam necessárias para a qualificação da $\mathrm{AB}$ no estado. No entanto, este profissional não possui autonomia para tomar decisões que facilitem os trâmites e as necessidades locais, cabendo aos gestores esta função.

Considerando os aspectos descritos até o momento, é possível perceber que o papel do articulador tem se construído de modo bastante abrangente, o que gera certa imprecisão e dúvidas por parte dos próprios profissionais a respeito de seu papel principal e de quais seriam as atividades de sua função.

\section{Atividades desempenhadas}

Devido ao papel de assessor das necessidades dos municípios em relação à qualificação da APS e à variação das mesmas, a definição de atividades mostrou-se uma tarefa árdua. Sendo assim, buscamos, através dos exemplos citados, definir algumas das atividades desenvolvidas pelos articuladores apresentadas no Quadro 2. Obviamente, estas não correspondem à totalidade, mas funcionam aqui como exemplos do que fazem e como fazem para cumprir os objetivos apresentados anteriormente.

A principal atividade inicial relatada se resumiu ao levantamento do diagnóstico sobre a 


2078 Quadro 2. Atividades desempenhadas pelos articuladores.
\begin{tabular}{l|l|}
\hline \multicolumn{1}{|c|}{ Tema } & \multicolumn{1}{c}{ Sentidos } \\
\hline $\begin{array}{l}\text { Atividades } \\
\text { desempenhadas }\end{array}$ & $\begin{array}{l}\text { - Realizar Diagnóstico; } \\
\text { - Avaliar possibilidade de implantação de novas propostas e programas; } \\
\text { - Participar das reuniões; } \\
\text { - Visitar os municípios; } \\
\text { - Repassar informações; } \\
\text { - Apoiar na confecção de documentos e no processo de implantação de algumas ações; } \\
\text { - Apoiar e orientar novos gestores. }\end{array}$ \\
\hline
\end{tabular}

organização da APS dos municípios através de entrevista direcionada e de consulta aos sistemas de informação. Fundamentados por este diagnóstico, os articuladores levantaram as necessidades específicas de cada município e fizeram propostas de melhoria, como a implantação de novos projetos, ou programas (propostos pelo Ministério da Saúde e, muitos deles, desconhecidos pelos gestores municipais) que fossem considerados úteis naquele momento ${ }^{4}$.

Conforme relato dos articuladores, no início do programa a Secretaria solicitava, via plataforma moodle ${ }^{14}$, atividades que deveriam ser cumpridas, como, por exemplo, o levantamento do diagnóstico e a implantação de novas propostas voltadas para as necessidades dos municípios. Porém, esse direcionamento se mostrava menor no ano de realização das entrevistas (2013). Consideramos que esta redução do acompanhamento pode gerar, muitas vezes, as dúvidas relatadas por eles quanto ao papel a ser desempenhado.

Os articuladores são, normalmente, convocados a participar de todas as reuniões sobre a $\mathrm{AB}$, como por exemplo: reuniões do colegiado; de equipes de educação permanente; da vigilância sanitária; vigilância epidemiológica; reuniões da SES, dentre várias outras. Em nossa análise, a participação das reuniões pode ser considerada uma das atividades que permite ao articulador exercer o seu papel de ampliador da comunicação, e acompanhar os municípios em sua organização, apoiando-os para "o desenvolvimento da capacidade de gestão municipal e da rede de Atenção Básica [...] mediante propostas de ações de intervenção e capacitação em Atenção Básica, pactuadas junto aos Municípios, Colegiados de Gestão Regional e Departamentos Regionais de Saúde"21.

Para que o articulador consiga fazer análises e propostas nestas reuniões, é necessário que ele conheça a realidade do município. Para isso, ele desempenha uma de suas atividades centrais, a visita aos municípios. Estas visitas se configuraram inicialmente como o foco de atuação do Programa. Em contato direto com os municípios, os articuladores são capazes de perceber as deficiências ou as necessidades de cada contexto, se estão relacionadas ao espaço físico; se relacionadas às relações (problemas de relacionamento entre profissionais, entre gestor e profissionais ou entre profissionais e usuários); à organização dos processos de trabalho (correspondência entre os papéis de cada um e sua boa execução); à gestão (problemas entre o que os gestores direcionam e o que os profissionais compreendem como sendo o papel deles) dentre outras possibilidades. Embora sejam de grande importância, a frequência das visitas diminuiu devido à demanda de trabalho administrativo do DRS.

Uma outra atividade se refere ao repasse de informações dos novos programas ou portarias lançados pelo Ministério da Saúde. Esse repasse pode ser realizado através do envio de e-mails; de conversas informais, ou através de treinamento solicitado por eles ao CDQ (Centro de Desenvolvimento e Qualificação). Esses treinamentos, eventualmente, são propostos e formulados pelos próprios articuladores de acordo com a necessidade e a disponibilidade de cada município.

E4: o Ministério da Saúde solta uma portaria, por exemplo, o PMAQ que é o Programa Nacional de Melhoria do Acesso à Qualidade da Atenção Básica, então qual que é o papel do articulador aí? É mostrar o passo a passo, é ir lá no município, incentivar a adesão, 'olha... o PMAQ é uma coisa boa...' eles vão avaliar, o município abre a porta pro Ministério da Saúde entrar, eles vão avaliar a equipe, mas a gente também tem que ver se o que a gente ta fazendo se ta certo, e... mas vocês vão ter um auxílio, vai ter um bônus aí, porque o PMAQ cai vinte por cento depois sessenta por cento, e no final cem por cento conforme o resultado. 
Os articuladores desempenham também atividade de apoio na confecção de documentos e do processo de implantação de algumas ações como: Plano Municipal de Saúde; Elaboração de Relatório Anual de Gestão; Acompanhamento dos indicadores de saúde e auxílio ao gestor no que concerne ao planejamento; Apoio no preenchimento do Sistema de Informação; Elaboração de projetos ou relatórios; Promoção de rodas de conversa; Orientação aos gestores com relação ao que negociar nas reuniões do colegiado, dentre outras.

A descrição acima, de algumas das atividades desenvolvidas pelos articuladores, sugere um acúmulo de responsabilidades e, muitas vezes, de sobrecarga de trabalho. Considerando este aspecto, questionamos os limites que esta sobrecarga pode representar à oferta de uma assessoria de qualidade, afinal, se o articulador deixa de visitar os municípios, ele perde seu principal recurso que é justamente a possibilidade de participar da realidade local. Poderíamos considerar que a redução das visitas constituiria uma descaracterização da função? Como articular, avaliar e monitorar a realidade local estando distante dos municípios?

No primeiro semestre de 2013, a principal atividade desenvolvida foi a de formação e acompanhamento dos novos gestores. As eleições haviam sido realizadas e os municípios estavam com novos prefeitos e novos secretários de saúde, ou seja, com novos horizontes de atuação política. Assim, os articuladores atuavam oferecendo auxílio para a formação desses políticos que em sua maioria não possuíam, segundo eles, conhecimento ou experiência na área da saúde.

A principal justificativa dada pelos articuladores para a falta de conhecimento dos secretários de saúde é o fato de ocuparem um cargo de natureza política. No entanto, consideramos que esta deveria ser a principal razão para se cobrar um conhecimento mínimo na área, afinal, este é um cargo de extrema responsabilidade estando a ele condicionada a organização e o modelo de atenção da saúde dos municípios, além do mais a literatura aponta que a ausência de conhecimento pode prejudicar o andamento das pactuações $^{12}$. Assim, a nova função do articulador acaba substituindo a gestão pouco instruída. Desse modo, o que deveria se constituir como assessoria pode, em alguns casos, a depender da região, funcionar como o papel do próprio gestor. Este tipo de situação deve ser analisado com cautela, afinal, sobrecarrega o articulador e exime o gestor de algumas responsabilidades.

Ao serem questionados sobre uma atuação voltada para gestão de conflitos interpessoais, os articuladores comentam perceber os conflitos como algo que permeia suas práticas, porém não reconhecem esta como sendo uma atividade própria da sua função, e chegam a comentar sobre um Programa recente, dos Articuladores da $\mathrm{Hu}-$ manização, que seria uma proposta para o cuidado dos aspectos micropolíticos.

As causas dos conflitos identificadas variam. Dentre elas estão: a falta de conhecimento com relação aos papéis de cada um; questões políticas como protecionismos dentro dos serviços; a presença do médico, apontada como uma das principais fontes de conflito, por ser ele o profissional com melhor salário e mais valorizado pela população, em contraste com o fato de ser o que trabalha menos horas; a mudança de gestão, devido aos novos direcionamentos ou devido à falta de confiança que estabelecem com profissionais escolhidos pela gestão anterior; e, falta de comunicação entre os profissionais, que, muitas vezes, não conseguem esclarecer problemas mínimos por não criarem espaços para o diálogo e o convívio das diferenças.

E3: existe, existe muito isso [refere-se a conflito]... [...] alguns profissionais... eles estão encaixados dentro de uma equipe de saúde por... por condições políticas.

Com relação à atuação nas situações que envolvem conflitos, os entrevistados compreendem como sendo necessária, principalmente quando notam o conflito atrapalhando o funcionamento geral dos serviços.

Portanto, embora mediar conflitos não seja uma atividade prevista, os articuladores reconhecem a importância das interações estabelecidas no cotidiano e de sua relação com a qualificação da AB. Como eles mesmos colocam, em alguns casos, este tipo de situação atrapalha a organização das unidades.

\section{Desafios}

São inúmeros os desafios citados pelos articuladores, muitos deles extrapolam as bases do Programa e se configuram como desafios da própria Reforma Sanitária do país, e das dificuldades enfrentadas para a consolidação da APS, conforme explorados na literatura e apontados na introdução, como a falta de recurso, os baixos salários, a alta rotatividade, dentre outros ${ }^{4-7,11-13}$. Considerando a diversidade dos desafios, os apresentamos organizados no Quadro 3.

$\mathrm{O}$ principal desafio se refere à mudança do modelo de atenção. Como colocado pelos articuladores, algo também amplamente discutido na 


\begin{tabular}{l} 
Quadro 3. Desafios da função do articulador da atenção básica. \\
$\qquad$\begin{tabular}{|l|l|}
\hline Tema & \multicolumn{1}{c|}{ Sentidos } \\
\hline $\begin{array}{ll}\text { Desafios da } \\
\text { prática }\end{array}$ & $\begin{array}{l}\text { - Estabelecer uma comunicação satisfatória entre instituições e atores; } \\
\text { - Negociar propostas mediante falta de recursos dos municípios; } \\
\text { - Organizar redes mediante falta de recursos físicos e humanos; } \\
\text { - Reorganizar processos de trabalho após mudanças de gestão; }\end{array}$ \\
- Sensibilizar profissionais de saúde, gestores e população quanto a um novo formato de \\
atuação não centrado na figura do médico; \\
- Reduzir a incidência das doenças crônicas (a equipe, muitas vezes, não atua com esse \\
objetivo); \\
- Auxiliar os municípios em suas dúvidas quanto a questões financeiras; \\
- Ter paciência para aguardar resultados quando o enfoque é prevenção e promoção de saúde; \\
- Lidar com equipes reduzidas e com a alta rotatividade; \\
- Resumindo, promover a mudança no modelo de atenção mediante falta de recursos.
\end{tabular} \\
\hline
\end{tabular}

literatura nacional e internacional ${ }^{4,5,13}$, o modelo de atenção na prática, muitas vezes, se mantém centrado na figura do médico e hospitalocêntrico. Este discurso permeia a atuação dos gestores, dos profissionais e da própria população que busca o serviço de saúde em momentos de crise, esperando receber consulta médica imediata.

A partir da enorme lista de desafios apresentados no quadro, consideramos que a própria manutenção do Programa se torna um desafio. Embora a proposta seja interessante, ela exige um acompanhamento constante, pois ao estar em contato com a realidade local, os articuladores têm condições de analisar quais aspectos das políticas e programas lançados em nível nacional deverão ser adaptados à realidade de cada contexto.

De acordo com os articuladores, apesar dos desafios, mudanças positivas podem ser observadas desde a implantação do Programa dentre as quais destacamos: melhoria do conhecimento dos profissionais e gestores a respeito das normas e programas; aumento de confiança dos gestores para tomar decisões; melhoria na qualidade da comunicação entre DRS e municípios; possibilidades de novos acordos entre entes federativos; abertura de espaço para que os profissionais possam se comunicar; melhoria significativa nos indicadores de saúde; inclusão da $\mathrm{AB}$ como pauta na reunião do colegiado de algumas regiões; melhoria na atualização dos sistemas de informação dentre outras.

Neste sentido, uma primeira análise do Programa e de suas características nos leva a sugerir que este pode ser um excelente recurso para promover a qualificação da $\mathrm{AB}$; por outro lado, a análise de como este Programa tem se construí- do no cotidiano das práticas, indica que as ações desenvolvidas pelos articuladores, muitas vezes, tornam-se específicas e de difícil gestão. Assim, como toda proposta de mudança, para que se desenvolva com efetividade, é necessário maior investimento e análise de seus resultados.

Mediante as conclusões da análise proposta, é importante ressaltar que este estudo possui um caráter inicial e exploratório a respeito de um Programa inovador do estado de São Paulo. Novos estudos, desenvolvidos com outros recortes metodológicos e perspectivas de análise podem complementar a por ora apresentada. Da mesma maneira, pode ser enriquecedor conhecer como articuladores das outras regionais do estado significam sua prática, permitindo aprofundar a compreensão sobre semelhanças e diferenças locais.

\section{Considerações finais}

A análise desenvolvida contribui para compreendermos o Programa dos articuladores, proposta recente da SES-SP e pouco divulgada. É possível notar com base nas informações descritas que o Programa possui potencial para qualificar a $A B$ e, ao mesmo tempo, para adequar as propostas nacionais à realidade local. Além disso, mostra-se como importante instrumento para o aumento da comunicação entre os entes federativos e entre os próprios profissionais da ponta do sistema, o que pode facilitar o trabalho intersetorial e a composição da rede.

Apesar de se constituir como importante recurso para a promoção de mudanças positivas, o Programa tem sido pouco divulgado, pouco es- 
tudado e pouco explorado, sendo vários os desafios destacados por esses novos profissionais que, muitas vezes, sentem-se desprovidos de recurso para lidar com as situações que encontram e para as quais são chamados a solucionar. É como se este Programa, criado para amenizar alguns desafios do contexto de consolidação da APS, se deparasse novamente com os mesmos desafios para os quais foi criado para amenizar.

Neste sentido, consideramos que o Programa sozinho não é capaz de promover transformações tão amplas pretendidas desde a Reforma Sanitária. A própria palavra Programa que o define, no contexto da saúde, carrega em si um sentido de transitoriedade, de algo criado com um fim específico e com ações pontuais previstas ${ }^{30}$. Sendo assim, questionamos: como amenizar os desafios enfrentados pelos articuladores e instrumentali- zá-los para que este trabalho, inicialmente focado, transitório, constituído como um Programa, transforme-se em uma estratégia, com sentido amplo e que inclua muitas frentes de ação?

A experiência dos articuladores da atenção básica pode contribuir em grande medida para o debate nacional, e até mesmo internacional, dos cuidados primários e da necessidade de adaptação às realidades locais e às transformações sociais constantes ${ }^{11-13}$. Se bem fundamentado, transformado e implementado pode contribuir para a construção da $\mathrm{AB}$ do país e, quem sabe no futuro, concretizar-se como uma experiência modelo para qualificação da APS. Mas, embora reconheçamos o seu potencial, não deixamos de observar que seu desenvolvimento envolve inúmeros desafios, alguns próprios do movimento contra-hegemônico da Reforma Sanitária.

\section{Colaboradores}

GC Doricci responsabilizou-se por todas as etapas da pesquisa, desde a realização das entrevistas, transcrições até a análise e a redação do material. C Guanaes-Lorenzi contribuiu com sua orientação em todas as etapas da pesquisa e de redação do artigo. MJB Pereira contribuiu para a discussão e a revisão teórica.

\section{Agradecimentos}

Agradecemos à Fundação de Amparo à Pesquisa do Estado de São Paulo (FAPESP).

\section{Referências}

1. Brasil. Portaria $n^{\circ} 2.488$, de 21 de outubro de 2011. Aprova a Política Nacional de Atenção Básica, estabelecendo a revisão de diretrizes e normas para a organização da Atenção Básica, para a Estratégia Saúde da Família (ESF) e o Programa de Agentes Comunitários de Saúde (PACS). Diário Oficial da União 2011; 22 out.

2. Organização Mundial de Saúde. Relatório Mundial de Saúde de 2008: Cuidados de Saúde Primários - Agora mais do que Nunca. Tradução. Genebra: OMS; 2008. [acessado 2015 dez 7]. Disponível em: http://www. who.int/whr/2008/whr08_pr.pdf

3. World Health Organization. Evaluation of the structure and provision of primary care in the republic of Moldova. A survey-based project. Primary Care in the WHO European Region. Denmark: Phoenix Design Aid, 2012. [acessado 2015 nov 10]. Disponível em: http://www. nivel.nl/sites/default/files/bestanden/Rapport-WHOMoldavie.pdf

4. Silveira CS, Machado CV, Matta GC. A Atenção Primária em Saúde na Agenda da Organização Pan-Americana de Saúde nos anos 2000. Trab. Educ. Saúde 2015; 13(1):31-44.

5. Mendes EV. A Construção Social da Atenção Primária à Saúde. Brasília: Conselho Nacional de Secretários de Saúde; 2015.

6. Mendes EV. As Redes de Atenção à Saúde: Revisão Bibliográfica, Fundamentos, Conceito e Elementos Constitutivos. In: Mendes EV, organizador. As Redes de Atenção à Saúde. Brasília: Organização Pan-Americana da Saúde; 2011. p. 61-208. 
7. Fertonani HP, Pires DEP, Biff D, Scherer MDA. Modelo assistencial em saúde: conceitos e desafios para a atenção básica brasileira. Cien Saude Colet 2015; 20(6):1869-1878.

8. Cavalcanti PCS, Oliveira Neto AV, Sousa MF. Quais são os desafios para a qualificação da Atenção Básica na visão dos gestores municipais? Saúde Debate 2015; 39(105):323-336.

9. Boerma WGW. Coordination and integration in European primary care. In: Saltman RS, Rico A, Boerma WGW, editors. Primary care in the driver's seat? Organizational reform in European primary care. Berkshire: Open University Press; 2007. p. 3-21.

10. Kringos DS, Boerma WGW, Hutchinson A, Van der Zee J, Groenewegen PP. The breadth of primary care: a systematic literature review of its core dimensions. $B M C$ Health Serv Res 2010; 0:65.

11. Santos AM, Giovanella L. Gestão do cuidado integral: estudo de caso em região de saúde da Bahia, Brasil. Cad Saude Publica 2016; 32(3):1-15.

12. Santos AM, Giovanella L. Governança regional: estratégias e disputas para gestão em saúde. Rev Saude Publica 2014; 48(4):622-631.

13. Almeida PF, Fausto MCR, Giovanella L. Fortalecimento da atenção primária à saúde: estratégia para potencializar a coordenação dos cuidados. Rev. Panam Salud Publica 2011; 29(2):84-95.

14. Andrade MC, Castanheira ERL. Cooperação e Apoio Técnico entre Estado e Municípios: a experiência do Programa Articuladores da Atenção Básica em São Paulo. Saúde Soc 2011; 20(4):980-990.

15. Mendes EV. Os Grandes Dilemas do SUS. Salvador: Casa da Qualidade; 2001. (Tomo II).

16. Mendes EV. O SUS que temos e o SUS que queremos. In: Conselho Nacional de Secretários Estaduais de Saúde (Conass). Convergências e divergências sobre gestão e regionalização do SUS. Brasília: Conass; 2004. p. 28-61.

17. Chebli ICF, Paiva MG, Stephan-Souza AI. A Regionalização: desdobramento da Descentralização? Rev. APS 2010; 13(Supl. 1):84-94.

18. Conselho Nacional de Secretários Estaduais de Saúde (Conass). Para entender o Pacto Pela Saúde 2006. Brasília: Conass; 2006.

19. São Paulo. Secretaria de Estado da Saúde de São Paulo. Pacto Pela Saúde. Plano Diretor de Regionalização PDR do Estado de São Paulo, 2008. [acessado 2012 abr 5]. Disponível em: http://portal.saude.gov.br/portal/ arquivos/pdf/pdr_sp.pdf

20. Governo do Estado de São Paulo. Secretaria de Estado da Saúde. [acessado 2011 dez 5]. Disponível em: http:// www.saude.sp.gov.br/ses/perfil/gestor/atencao-basica/ Programa-articuladores-de-atencao-basica/articuladores-da-atencao-basica
21. São Paulo (estado). Resolução SS no 61 , de 14 de Junho de 2011. Diário Oficial do Estado de São Paulo 2011; 15 jun.

22. McNamee S. Construindo conhecimento / construindo investigação: coordenando mundos de pesquisa. In: Guanaes-Lorenzi C, Moscheta MS, Corradi-Webster $\mathrm{CM}$, Souza LV, organizadores. Construcionismo Social: discurso, prática e produção do conhecimento. Rio de Janeiro: Instituto Noos; 2014. p.105-131.

23. McNamee S. Research as social construction: transformative inquiry. Saúde Transform. Soc., 1(1):09-19, 2010.

24. Spink MJP, organizador. Práticas discursivas e produção de sentidos no cotidiano: aproximações teóricas e metodológica. 3a ed. São Paulo: Cortez; 2004.

25. Spink MJP, Medrado B. Produção de sentidos no cotidiano: uma abordagem teórico-metodológica para análise das práticas discursivas. In: Spink MJP, organizador. Práticas discursivas e produção de sentidos no cotidiano: aproximações teóricas e metodológicas. $3^{\mathrm{a}} \mathrm{ed}$. São Paulo: Cortez; 2004. p. 41-62

26. Guanaes C. A Construção da mudança em Terapia de Grupo: um enfoque construcionista social. São Paulo: Vetor; 2006

27. Preti D. Análise de textos orais. $4^{\mathrm{a}}$ ed. São Paulo: Humanitas Publicações FFCLRP, USP; 1999. (Série projetos paralelos v.1).

28. Spink MJP, Lima H. Rigor e visibilidade: a explicitação dos passos da interpretação. In: Spink MJP, organizador. Práticas discursivas e produção de sentidos no cotidiano: aproximações teóricas e metodológicas. $3^{\mathrm{a}}$ ed. São Paulo: Cortez; 2004. p. 93-122.

29. Rodrigues LBB, Silva PCS, Peruhype RC, Palha PF, Popolin MP, Crispim JA, Pinto IC, Monroe AA, Arcêncio RA. A atenção primária à saúde na coordenação das redes de atenção: uma revisão integrativa. Cien Saude Colet 2014; 19(2):343-352.

30. Corbo AD’A, Morosini MVGC, Pontes ALM. Saúde da Família: construção de uma estratégia de atenção à saúde. In: Morosini MVGC, Corbo AD’A. Modelos de Atenção e a Saúde da Família. Rio de Janeiro: EPSJV, Fiocruz; 2007

Artigo apresentado em 20/01/2016

Aprovado em 16/08/2016

Versão final apresentada em 18/08/2016 